\title{
Do Auto-Regressive Models Protect Privacy? INFERRING FINE-GRAINED ENERGY CONSUMPTION FROM Aggregated Model Parameters
}

\author{
A PREPRINT
}

\author{
(1) Nazim Uddin Sheikh* \\ Department of Computing \\ Macquarie University \\ Sydney, Australia \\ nazim-uddin.sheikh@hdr.mq.edu.au \\ ๑ Farhad Farokhi \\ University of Melbourne \\ Melbourne, Australia
}

\author{
(1) Hassan Jameel Asghar \\ Department of Computing \\ Macquarie University and Data61, CSIRO \\ Sydney, Australia
}

\author{
- Mohamed Ali Kaafar \\ Department of Computing \\ Macquarie University \\ Sydney, Australia
}

August 6, 2021

\begin{abstract}
We investigate the extent to which statistical predictive models leak information about their training data. More specifically, based on the use case of household (electrical) energy consumption, we evaluate whether white-box access to auto-regressive (AR) models trained on such data together with background information, such as household energy data aggregates (e.g., monthly billing information) and publicly-available weather data, can lead to inferring fine-grained energy data of any particular household. We construct two adversarial models aiming to infer fine-grained energy consumption patterns. Both threat models use monthly billing information of target households. The second adversary has access to the AR model for a cluster of households containing the target household. Using two real-world energy datasets, we demonstrate that this adversary can apply maximum a posteriori estimation to reconstruct daily consumption of target households with significantly lower error than the first adversary, which serves as a baseline. Such fine-grained data can essentially expose private information, such as occupancy levels. Finally, we use differential privacy (DP) to alleviate the privacy concerns of the adversary in dis-aggregating energy data. Our evaluations show that differentially private model parameters offer strong privacy protection against the adversary with moderate utility, captured in terms of model fitness to the cluster.
\end{abstract}

Keywords aggregate statistics - white-box attacks · inference attacks · auto-regressive models · differential privacy · energy data privacy.

\section{Introduction}

Smart meters, an integral component of the smart grids infrastructure, are now widely deployed by electricity providers and retailers to monitor nearly in real time fine-grained energy consumption of households. The measurements, often communicated to the service providers over a secure channel [Erkin et al., 2013], are used for grids monitoring and management [Knirsch et al., 2016].

\footnotetext{
*The article has been accepted at IEEE Transactions on Services Computing in July 2021.
} 
Smart-meters data can also be used to forecast electricity consumption in the form of statistical predictive models, for example, auto-regressive (AR) models, built over a collection (cluster) of households with similar electricity usage [Dagnely et al., 2015]. These models can provide insights for grid provisioning, for example, in high consumption periods such as summer, and assessment of economic and environmental impact. Thus, sharing such models has great societal benefits. A key concern is, however, the privacy of the underlying households. For instance, it has been shown that if fine-grained energy consumption of a household is known then detailed information about its inhabitants, such as daily routines and household occupancy can be inferred [Molina-Markham et al., 2010, Chen et al., 2015, Eibl and Engel, 2014].

\subsection{Motivation}

Machine learning (ML) algorithms and statistical models (e.g., auto-regressive model and it's variants) are widely being used for forecasting and analysing various user-centric data, such as electricity usage of households [Nichiforov et al., 2017, Alden et al., 2020, Shah et al., 2020, Vu et al., 2019, Xiao et al., 2018, Kaytez, 2020, Guefano et al., 2021]. Statistical models are much simpler than ML algorithms and can still provide an efficient and effective way to facilitate skillful single-step and multi-step predictions for electricity consumption [Ruiz et al., 2016, Dambreville et al., 2014, Singh and Pozo, 2019]. Further, sharing and reusing such pre-trained models can be beneficiary for business developments [Avsec, 2018]. In particular, the increasing use, share and reuse of statistical and ML models can significantly simplify and expedite the development cycles of machine learning as a service (MLaaS), statistical model as a service (SMaaS) and hybrid services ${ }^{2}$ on the cloud [Wu et al., 2020, Hossain et al., 2019, AlShehhi and Wang, 2020]. However, sharing and reusing such models may cause profound privacy implications on the privacy of users if the models are trained on user-centric data [Ji et al., 2018].

This motivates us to study the impact of model sharing on the privacy of energy consumers. More specifically, an important question is whether it is possible to infer fine-grained energy consumption of a household whose data was used to construct simple statistical models; in particular, in the presence of background information about the target households. An example of such background information is the aggregated energy usage data available through monthly or quarterly electricity bills. Access to the latter is relatively easy for an adversary, for instance, via mail theft [Hedayati, 2012], trash theft [Philpott, 2006, Hedayati, 2012], or by collusion between marketing agencies and banks. On the other hand, statistical models can be widely shared for forecasting and extensive data analyses. Similar to the use of MLaaS, many technology companies have already started providing MLaaS platforms with support for statistical models for commercial use. For instance, Google Inc.'s Cloud ML Engine ${ }^{3}$, Amazon AWS's SageMaker ${ }^{4}$ BigML $^{5}$ and Microsoft Corporation's Azure ${ }^{6}$ provide a wide range of ML algorithms, statistical models ${ }^{7}$ and hybrid models (e.g., DeepAR ${ }^{8}$ ). The next generation MLaaS systems, such as BigML and Azure Learning, facilitate a white-box access of pre-trained models through an API with the consent of the models or data owners[Fredrikson et al., 2015]. Moreover, there are some open source MLaaS or SMaaS platforms, such as OpenML [Van Rijn et al., 2013], ModelDB [McDougal et al., 2017] and Acumos [Zhao et al., 2018], which allow the sharing of pre-trained models and the model source code. Using these platforms data scientists can use their data to train a ML or statistical models for prediction and classification purposes. Subsequently, the data and model owners, influenced by financial incentives, may allow access to other application service providers or end-users to expedite application development [Zhao et al., 2018] for further business analysis, research, societal and commercial benefits. Thus, data owners or owners of trained models give white-box access [Shokri et al., 2017] to the model parameters trained on aggregated data which would help third-parties build applications based on forecasts from the model. Unfortunately, the aggregated nature of the data used for training the model parameters is often constructed and considered to be providing adequate privacy.

It has previously been shown that having (white-box) access to publicly available ML models and some additional background knowledge about the target users, a potential attacker is capable of exposing sensitive information or pursuing membership inference attack [Shokri et al., 2017] by exploiting the statistical correlation (of training dataset) encoded in the parameters of the model [Wu et al., 2016]. In our setting, we consider an adversary, with white-box access to a simple linear statistical model (auto-regressive exogenous or ARX model is chosen for the simplification

\footnotetext{
${ }^{2}$ Statistical models integrated with ML or deep learning models.

${ }^{3}$ https://cloud.google.com/ai-platform

${ }^{4}$ https://aws.amazon.com/sagemaker/

${ }^{5}$ https://www.bigml.com/

${ }^{6}$ https://azure.microsoft.com/en-au/services/machine-learning/

${ }^{7}$ https://docs.aws.amazon.com/forecast/latest/dg/aws-forecast-recipe-arima.html

${ }^{8}$ https://docs.aws.amazon.com/sagemaker/latest/dg/deepar.html
} 
of the attack model) and having some additional knowledge (monthly energy bill) of the target households, is capable of reconstructing the fine-grained energy consumption data of the target users.

\subsection{Contributions}

In this paper we aim to (i) quantitatively investigate the privacy implications of releasing statistical predictive models while having access to monthly aggregated energy consumption of households as background knowledge, and (ii) assess the efficiency of DP [Dwork and Roth, 2014] as a privacy-preserving mechanism to prevent inference of finegrained (daily) energy consumption. Our contributions are as follows:

- We construct two adversarial models depending on the information available to the adversary. The first adversary $\mathcal{A}_{1}$ serves as a baseline and is only provided with monthly aggregates of a target household. The second adversary $\mathcal{A}_{2}$ has also access to the parameters of a statistical model, specifically, an AR model, trained on the mean energy consumption of a cluster of households which includes the target household, in addition to the monthly aggregates. We evaluate the ability of these adversaries in estimating fine-grained, i.e., daily, household consumption by using a maximum a posteriori (MAP) estimation [Gauvain and Lee, 1994].

- Using two real-world energy usage datasets we show that having access to model parameters and monthly consumption aggregates enables an adversary to construct fine-grained consumption of target households. In particular, we show that an adversary who has access to the model parameters is able to infer $90 \%$ of the daily energy consumption time-series with 2 to 5 times less absolute error than an adversary provided only with monthly aggregates; see Section 4 . Furthermore, the adversary can predict daily peaks and lows of a target household even if they deviate from the mean consumption of the corresponding cluster. The accuracy of inference is dependent on how well the model fits to the mean energy consumption of the cluster.

- To alleviate the privacy concerns raised in the analysis, we use DP [Dwork and Roth, 2014] to perturb the mean daily energy consumption of a cluster of households before training the model. We show that DP can indeed prevent reconstruction of fine-grained energy consumption data from the aggregates while providing good utility in terms of model fitness. With a privacy budget of $\epsilon=0.1$, we achieve nearly $70 \%$ model fitness with the second adversary's error profile degrading to that of the baseline; see Section 5.

\section{Related Work}

In this section, we review some related work pertaining to adversarial attacks against smart meter data and the impact of such attacks on the privacy of electricity consumers. Moreover, we discuss some related works that focus on differential privacy to minimise the privacy leakage in smart meter data.

We discuss some potential attack models that focus on how the privacy of customers can be breached from their time series and aggregated [Tudor et al., 2013, Bohli et al., 2010, Buescher et al., 2017, Kelly and Knottenbelt, 2015, Karim et al., 2020] and masked [Barbosa et al., 2014, Baloglu and Demir, 2018] energy consumption data. Bohli et al. [Bohli et al., 2010] presented a cryptographic game-based adversarial model to measure the degree of privacy that a smart metering application can provide. Tudor et al. [Tudor et al., 2013] constructed an attack framework to de-anonymise customers' identity in a big database by linking low granular data with high granular smartmeter readings. Further, a UK-based industry body studied that an aggregation of two smart-meter readings is sufficient to protect individuals from being identified [ena, 2015]. However, the report in [ena, 2015] was challenged by Buescher et al. [Buescher et al., 2017] by demonstrating that the privacy implications may vary with the size of aggregation groups concluding that a group size of two in an aggregation is potentially inadequate to protect privacy. Moreover, a plethora of energy disaggregation mechanisms have been proposed using Markov models [Makonin et al., 2015, Guo et al., 2014, Kong et al., 2016], supervised ML models [Beckel et al., 2014], deep learning models [Kelly and Knottenbelt, 2015]. These disaggregation techniques could be used to infer appliance specific load patterns from aggregated energy data [McLaughlin et al., 2011].

Karim et al. [Karim et al., 2020] proposed an attack framework to perform white-box and black-box attacks against various time series data using machine learning models and also envisaged some defense mechanisms. Further, model inversion attacks [Fredrikson et al., 2015, Fredrikson et al., 2014, Yang et al., 2020, Wu et al., 2016] have been put forward against ML models to infer sensitive information. Furthermore, membership inference attacks [Shokri et al., 2017, Pyrgelis et al., 2017] have been well studied to infer the membership of a user in an aggregated and training data of a variety of ML models. These attacks can be performed using both white-box [Sablayrolles et al., 2019, Hayes et al., 2019, Nasr et al., 2018] and black-box [Sablayrolles et al., 2019, Hayes et al., 2019, Papernot et al., 2017] access to the pre-trained models. 
The above discussed attack models and energy disaggregation techniques induce an explicit violation of the privacy of consumers since private information, such as occupancy level, daily routine and home activities can be inferred [Molina-Markham et al., 2010, McLaughlin et al., 2011]. Moreover, these attack models presented privacy concerns, such as determining personal behavior patterns (which can be potentially used by the governments or marketing companies) and occupancy levels (for home invasion by criminals), when deploying a smart-meter infrastructure collecting fine-grained energy consumption of consumers [Lisovich et al., 2010, Quinn, 2009, Bohli et al., 2010, Saputro and Akkaya, 2014]. Our adversarial model focuses on white-box attack using a simple statistical model by reverse-engineering its mathematical structure and using monthly aggregated bills of users.

Finally, we review the effect of DP [Dwork et al., 2017, Dwork and Roth, 2014] in protecting the privacy of smart meters data [Chatzikokolakis et al., 2013, Giaconi et al., 2020]. A myriad of works [Eibl and Engel, 2017, Ács and Castelluccia, 2011, Zhao et al., 2014, Liao et al., 2017, Barbosa et al., 2016, Mak et al., 2019, Yang et al., 2017] studied the effect of DP on aggregated energy data in protecting privacy of users while collecting the fine-grained meter readings. Another privacy-preserving protocol using DP was presented in [Danezis et al., 2011] by adding noise to the aggregate energy consumption (bills) of consumers to hide their activities that might be revealed from their energy consumption traces. Furthermore, Liao et al. [Liao et al., 2014] proposed a privacy preserving energy data analytics framework using distributed DP. However, we focus on perturbing a predictive model (by perturbing the input time series used for training the predictive models) via the Laplace [Dwork, 2011] and Gaussian [Dwork et al., 2006a] mechanisms to mitigate the reconstruction attack. Thus, the model parameters are private due to post-processing property of DP. Further, we show how to achieve better utility in terms of model fitness whereas, the existing literature focused on the utility of the aggregated energy data; see Section 5 .

\section{Energy Data Models and Inference}

We define a trusted data curator $\mathcal{C}$, which abstractly plays the role of energy retailers or producers. In particular, $\mathcal{C}$ is in possession of fine-grained household consumption, monthly aggregates, and parameters of the predictive models.

\subsection{Mathematical Preliminaries}

\subsubsection{Notation}

The energy reading of household $i$ by a smart meter at time step $t \in \mathbb{N}$ is denoted by $x_{i}(t) .{ }^{9}$ Energy data of household $i$ over horizon $T$ (i.e., $T$ time steps) is represented as a time-series, and denoted by the $T$-element vector $\mathbf{x}_{i}$, whose $t$-th element is $x_{i}(t)$. The aggregate consumption of household $i$ over a time period $\Delta t$, defined as a set of one or more time steps, is given by

$$
y_{i}(\Delta t)=\sum_{t \in \Delta t} x_{i}(t) .
$$

We consider $\Delta t$ to be a monthly period, and hence $y_{i}(\Delta t)$ would denote aggregate for the corresponding month. Aggregate consumption over multiple time periods for household $i$ will be denoted by $\mathbf{y}_{i}$. When talking about a generic household, we will drop the subscript $i$, and simply write $x(t), \mathbf{x}$, or $\mathbf{y}$.

\subsubsection{Auto-Regressive Models.}

Auto-Regressive (AR) models are common statistical models for filtering and predicting time series data, analyzing their behavior, and detecting anomalies [Box et al., 2015]. AR refers to a stochastic process in which the current output depends linearly on previous outputs. An AR model of $\operatorname{order}^{10} n_{h}$, denoted by $\operatorname{AR}\left(n_{h}\right)$, is defined as

$$
x_{t}=\sum_{k=1}^{n_{h}} h_{k} x_{t-k}+w_{t},
$$

where integer $t$ denotes a time step, $x_{t} \in \mathbb{R}$ is the output of the AR model, and $w_{t} \in \mathbb{R}$ is the process noise, often assumed to be a zero-mean Gaussian variable with standard deviation $\sigma$.

\footnotetext{
${ }^{9}$ In general, time steps $t$ and $t+1$ represent consecutive, potentially equally-spaced, times. In this paper, they represent days.

${ }^{10}$ The order of an AR model $n_{h}$ captures its memory length, i.e., the number of previous outputs on which the current output depends.
} 
Models can sometimes depend on outside factors, known as exogenous variables or inputs. For energy consumption data this could be daily average temperature. An AR model with exogenous input, generally denoted by ARX [Galrinho et al., 2017], is described by

$$
x(t)=\sum_{k=1}^{n_{b}} b_{k} u_{t-k}-\sum_{k=1}^{n_{h}} h_{k} x_{t-k}+w_{t},
$$

where $u_{t} \in \mathbb{R}$ is the exogenous input, $1 \leq n_{b} \leq n_{h}<T$, and $t \in\left\{n_{h}+1, \ldots, T\right\}$. We set that $n_{b}=n_{h}$ throughout this paper. When modelling energy data time series, it is assumed that $x_{t}$ follows an ARX model. Over the horizon $\{1,2, \ldots, T\}$.

The generalised ARX model can be rewritten in matrix form as

$$
H \mathbf{x}=B \mathbf{u}+\mathbf{w}
$$

where, $\mathbf{x}, \mathbf{u}$ and $\mathbf{w}$ are column vectors representing energy readings, daily temperature data (as exogenous inputs) and noise, respectively, over the horizon $T$, and $H$ and $B$ are matrices representing the model parameters $b_{k}$ 's and $h_{k}$ 's, respectively. Note that the above matrices are represented as the the Hankel matrices or the transpose of the Toeplitz matrices (aka diagonal-constant matrix) in which each descending diagonal from left to right is constant. (see the Appendix for detail explanation of the generalised matrices).

Statistical properties of $\mathbf{w}=H \mathbf{x}-B \mathbf{u}$ determine if an AR model fits the data or not. For example, we may use a hypothesis testing mechanism to determine if $\mathbf{w}$ is Gaussian distributed with a given mean and variance. The confidence of such a test can serve as a measure of the fitness of the model. This is similar to the Dickey-Fuller test [Paparoditis and Politis, 2018] but over a fixed horizon rather than a rolling window.

Example: We demonstrate the AR model using a toy example for a small number of data points. Let us consider a time series $\mathbf{x}=\left[x_{0}, x_{1}, x_{2}, x_{3}, x_{4}\right]=[2,3,1,2,1]$ with five time steps (i.e., $t=5$ ) and AR model order be $n_{h}=1$. Then, we estimate the next time step $x_{5}$ using AR model defined in Equation (2) as follows.

$$
\begin{array}{r}
x_{5}=h_{1} x_{4}+w_{5} \\
=1 h_{1}+w(5)
\end{array}
$$

where, $w(5)$ is the white-noise and all the coefficients $\left(h_{1}\right)$ are computed using the correlation formula. We can form the over-determined system as follows.

$$
\left[\begin{array}{l}
3 \\
1 \\
2 \\
1
\end{array}\right]=\left[\begin{array}{l}
2 \\
3 \\
1 \\
2
\end{array}\right] h_{1},
$$

where,

$$
\mathbf{P}=\left[\begin{array}{l}
3 \\
1 \\
2 \\
1
\end{array}\right], \mathbf{Q}=\left[\begin{array}{l}
2 \\
3 \\
1 \\
2
\end{array}\right]
$$

and this can be solved using least-square estimator.

$$
\hat{h}_{1}=\left(\mathbf{P}^{\mathbf{T}} \mathbf{P}\right)^{-\mathbf{1}} \mathbf{P}^{\mathbf{T}} \mathbf{Q}=\frac{\sum_{t=0}^{3} x_{t-1} x_{t}}{\sum_{t=0}^{3} x_{t-1}^{2}}=\frac{c_{1}}{c_{2}}=r_{1},
$$

where, $c_{i}$ and $r_{i}$ are the $i^{t h}$ autocovariance and autocorrelation coefficients, respectively. An adversary acquires the model parameters (i.e., $h_{1}$ in the examples) then attempts to reconstruct the actual time-series $\mathbf{x}$ with some background knowledge by reverse-engineering the model structure.

\subsubsection{Maximum a Posterior (MAP) Estimation}

We are interested in knowing if auxiliary information in the form of aggregate monthly statistics and ARX model parameters, could lead to inference of fine-grained time series x. For a given household, we model aggregate consumption over the horizon as a vector $\mathbf{y}$ defined over $m$ time intervals, given as

$$
\mathbf{y}:=\left[\begin{array}{llll}
y\left(\Delta t_{1}\right) & y\left(\Delta t_{2}\right) & \ldots & y\left(\Delta t_{m}\right)
\end{array}\right]^{\top},
$$


where the union of the disjoint time intervals $\Delta t_{i}$ equals the set $\{1, \ldots, T\}$. As mentioned before, each $\Delta t_{i}$ spans a month. There exists a matrix $M \in \mathbb{R}^{m \times T}$ such that

$$
\mathbf{y}=M \mathbf{x},
$$

where $\mathbf{x}$ is the time-series for the corresponding household. The matrix $M$ is such that its $i$-th row has ones in locations corresponding to the interval $\Delta t_{i}$ and zeros in all other locations. This is public information (e.g., the billing cycle is known publicly).

The other piece of auxiliary information is the ARX model parameters, i.e., the matrices $H$ and $B$, and exogenous input $\mathbf{u}$ as defined in Eq. (4). In this case we can define the problem of inferring $\mathbf{x}$ as a maximum posterior estimation problem. We are given $\mathbf{y}, H, B$, and $\mathbf{u}$. Assuming that the elements of $\mathbf{w}$, in Eq. (4), are independently and identically distributed (i.i.d.) Gaussian random variables with zero mean and variance $\sigma$, we can deduce that the posterior probability follows

$$
p(\mathbf{x} \mid H, B, \mathbf{u}) \propto \exp \left(-\frac{1}{2 \sigma}\|H \mathbf{x}-B \mathbf{u}\|_{2}^{2}\right) .
$$

Thus, a MAP estimate of $\mathbf{x}$, denoted by $\hat{\mathbf{x}}$, can be obtained by solving the following optimization problem

$$
\begin{array}{cl}
\hat{\mathbf{x}} \in \underset{\mathbf{x}}{\operatorname{argmin}} & \|H \mathbf{x}-B \mathbf{u}\|_{2}^{2}, \\
\text { s.t. } & M \mathbf{x}=\mathbf{y} .
\end{array}
$$

We consider the case where the ARX model is constructed using the mean daily time-series of a cluster of (similar) households.

If the ARX model is unknown, by assuming $\mathrm{x}$ is an i.i.d. zero-mean Gaussian variable, we can obtain an estimate by

$$
\begin{aligned}
\hat{\mathbf{x}} \in \underset{\mathbf{x}}{\operatorname{argmin}} & \|\mathbf{x}\|_{2}^{2}, \\
\text { s.t. } & M \mathbf{x}=\mathbf{y} .
\end{aligned}
$$

\subsection{Energy Estimation as an Inference Problem}

We model the inference of fine-grained energy data as a game played between an adversary $\mathcal{A}$ and the curator $\mathcal{C}$. The adversary first chooses a target household, followed by the curator sending background information about the household to the adversary. This models the background information known to the adversary. The game concludes with the adversary submitting its estimate of the fine-grained time series. The success of the adversary is assessed by the error in estimating the time series. Depending on the background information provided, we define two different adversaries, $\mathcal{A}_{1}$ and $\mathcal{A}_{2}$ (and hence two different games) explained below.

Adversary $\mathcal{A}_{1}$ (Baseline) This models an adversary with only access to monthly aggregate information about a target household, and serves as a baseline. As mentioned in the introduction, this information can be obtained in a variety of ways, e.g., following a public data release of aggregate monthly consumption of households by the government or a service provider for business analytics and research purposes or from the utility bills which can be obtained via mail theft [Hedayati, 2012], trash theft [Philpott, 2006, Hedayati, 2012], or through collusion between marketing agencies and banks. In the game played between $\mathcal{A}_{1}$ and $\mathcal{C}$, the adversary queries the curator for accessing the aggregate monthly consumption $\mathbf{y}_{i}$ of a target household $i$; see Figure 1. Using this aggregate information, $\mathcal{A}_{1}$ attempts to estimate $\mathbf{x}$ using the MAP estimate in Eq. (8). The adversary submits its estimate $\hat{\mathbf{x}}_{i}$ to $\mathcal{C}$. At this stage, the curator computes the absolute squared error (SE) between $\mathbf{x}_{i}$ and $\hat{\mathbf{x}}_{i}$ to measure $\mathcal{A}_{1}$ 's success, defined as:

$$
\mathrm{SE}_{i}=\left[\begin{array}{c}
\left|x_{i}(1)-\hat{x}_{i}(1)\right|^{2} \\
\vdots \\
\left|x_{i}(T)-\hat{x}_{i}(T)\right|^{2}
\end{array}\right]
$$

Adversary $\mathcal{A}_{2}$ In this adversarial setting, we set that in addition to the monthly aggregates of a target household, the adversary has the parameters of an ARX model trained on average energy consumption time-series data $(\mathrm{x})$ of a cluster of households (including the target household). $\mathcal{A}_{2}$ can obtain the monthly aggregates (bills) from the similar sources as $\mathcal{A}_{1}$ obtains as discussed above. 


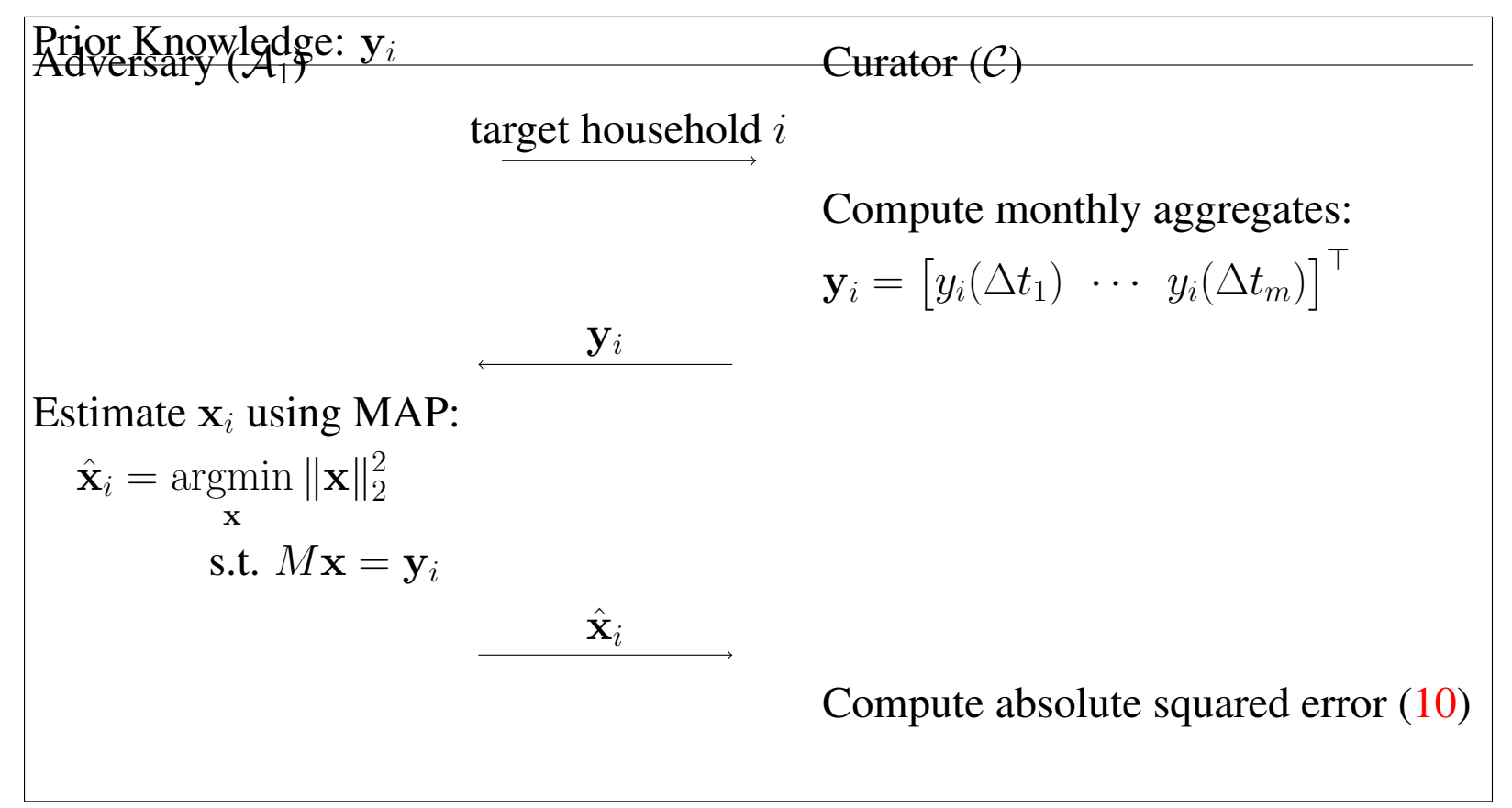

Figure 1: Estimation of energy consumption as an inference game played between $\mathcal{A}_{1}$ and a curator $\mathcal{C}$.

We now depict a realistic scenario that how an adversary can have white-box access to the parameters of statistical models, e.g., auto-regressive model. Acumos, an open source MLaaS/SMaaS, is being as common as commercial MLaaS used for predicting sequential data. Acumos Platform has a provision of publishing models to expedite application development [Zhao et al., 2018], for further business analysis, prediction, research, societal and commercial benefits. In this setting or in a similar setting, we consider a utility provider or a data owner or a curator may use such open source platform to train their consumers' data as a cluster or aggregated data into a model for forecasting and/or for further analyzing their data without any expertise in statistical models such as AR model which is in our case, subsequently, publishes the model parameters. A malicious user (e.g., a marketing company) who is not authorised to observe the training data of the data holder but can have white-box access to the resulting model. Then, the adversary suspects that her target household's energy data is used to train the model. In this setting, a malicious user ( $\mathcal{A}_{2}$ in our setting) with white-box access to the auto-regressive model may be able to reconstruct the training data by reverse-engineering the model.

More precisely, the data owner or model owner (i.e., energy provider) gives white-box access of the model to other users or releases the model parameters $H$ and $B$ defined in Eq. (4) for further forecasting in business analysis and research purposes through an open source platform. Using this information, the adversary (a malicious user) attempts to estimate $\mathbf{x}_{i}$ based on the MAP approach defined in Eq. (7). At this stage, the curator computes the absolute squared error (SE) between $\mathbf{x}_{i}$ and $\hat{\mathbf{x}}_{i}$ to measure $\mathcal{A}_{2}$ 's success, as defined in Eq. (9).

We demonstrate the energy estimation cryptograhic game-based approach inspired by other works in [Bohli et al., 2010, Buescher et al., 2017] using an example. Let us suppose, Alice, a data owner, trains a statistical/ML model on a cloud-based platform using an aggregated consumption of a group of households' daily energy consumption with similar patterns to gain a deeper inception and for forecasting purpose. Then, she gives a white-box access to the parameters of her pre-trained models for research and societal benefit or influenced by incentives. On the other hand, Bob, an end-user on the cloud platform, attempts to infer individual consumption by using these parameters along with some background information, such as the monthly consumption of the target households. 


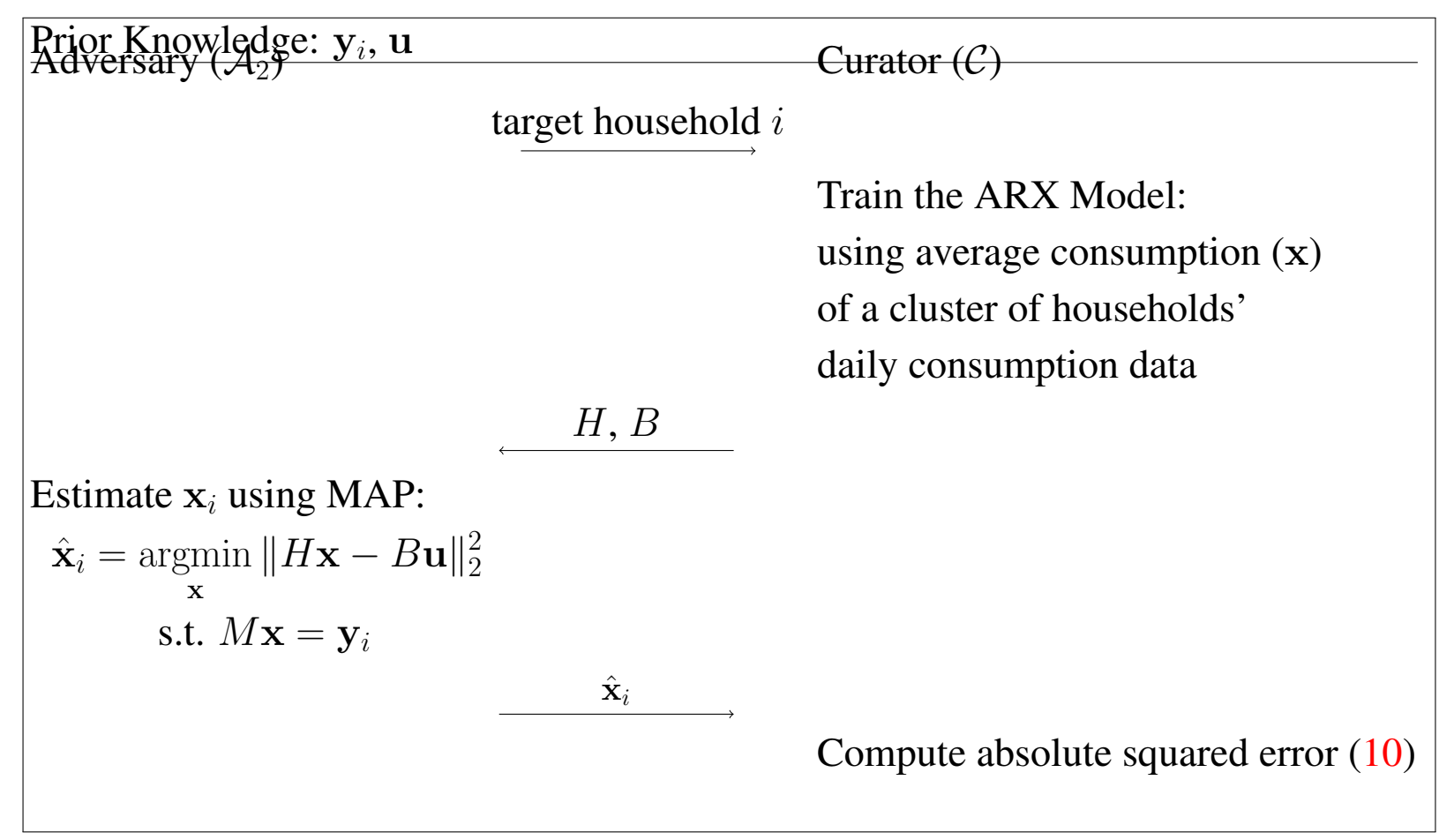

Figure 2: Estimation of energy consumption as an inference game played between $\mathcal{A}_{2}$ and a curator $\mathcal{C}$.

\begin{tabular}{lclclc}
\hline Dataset Name & Geographic Location & Year & Meter Reading Interval & Number of Households & Weather Data \\
\hline UMass & US & 2015,2016 & $15 \mathrm{~min}, 1 \mathrm{~min}$ & 114 & Daily Temperature \\
UK Power Networks & London, UK & 2013 & $30 \mathrm{~min}$ & 4681 & Daily Temperature \\
\hline
\end{tabular}

Table 1: Some features of the energy datasets and daily temperature data (exogenous input) used in the experiments are summarized.

\subsection{Datasets}

We use two real-world datasets that capture the energy-consumption records of households, obtained, respectively, from the UMass Trace Repository ${ }^{11}$ which also contains the weather data of the particular region and the UK Power Networks ${ }^{12}$. We also collect the weather data (daily temperature) from the Dark Sky API ${ }^{13}$. These open-source datasets contain different numbers of households with a variety of smart-meter reading frequency over various periods of time. The characteristics of the datasets are summarized in Table 1.

\subsection{Experimental Setup}

First we use K-Means clustering [Green et al., 2014] to divide the datasets into $K$ clusters with similar energy consumption patterns among the households. We set $K=2$ and $K=9$ (which means that we construct 2 and 9 clusters) for UMass (of the year 2015 and 2016) and London-2013 (of the year 2013 obtained from the UK Power Networks) datasets, respectively. Determining the optimal number of clusters in a dataset is a fundamental problem in clustering algorithms. We choose the number of clusters based on Silhouette score [Hotho et al., 2002] which is a measure of how similar the energy consumption pattern of a household to other households in its own cluster compared to other clusters. Setting these values of $K$, we observe high Silhouette scores which render good fitness whereas further increasing the values of $K$ Silhouette scores either declines or remains very similar to its previous scores. We use sklearn ${ }^{14}$ to implement the K-Means clustering algorithm on the aforementioned datasets.

\footnotetext{
${ }^{11}$ See http://traces.cs.umass.edu/index.php/Smart/Smart

${ }^{12}$ See https://data.london.gov.uk/dataset

${ }^{13}$ See www. darksky . net/dev

${ }^{14}$ See https://scikit-learn.org/stable/modules/clustering.html
} 
We use the arx function in MATLAB to train ARX models for each time-series ${ }^{15}$. We set $n_{h}=n_{b}=5$ in Eq. (3) to obtain a good fitness of the model. We further use the arxOptions function to set the initial condition to 'auto', meaning that we let the function decide whether to set the initial conditions to zero or to treat them as independent parameters. Note that the first $n_{h}$ readings of a time series constitute its initial conditions, which are not predicted by the ARX model in Eq. (3). In return, we get a set of parameters for the ARX model.

To obtain MAP estimates for the adversaries, we use the MATLAB-based CVX toolbox ${ }^{16}$, which allows specifying convex optimization problems using standard MATLAB syntax and solved by off-the-shelf solvers.

\subsection{Evaluation Metrics}

Overall Performance. The overall accuracy of an adversary's estimation is assessed using absolute mean squared error (MSE) over all households in a cluster in a given dataset. Specifically, MSE is defined as

$$
\operatorname{MSE}:=\frac{1}{n}\left[\begin{array}{c}
\sum_{i=1}^{n}\left|x_{i}(1)-\hat{x}_{i}(1)\right|^{2} \\
\vdots \\
\sum_{i=1}^{n}\left|x_{i}(T)-\hat{x}_{i}(T)\right|^{2}
\end{array}\right],
$$

where $n$ is the number of households in a cluster and $x_{i}(j)$ and $\hat{x}_{i}(j)$ refer to the actual and estimated daily consumption of a household $i$ at time step $j$, respectively. Thus, we get a vector of error values over the entire horizon $T$ for each cluster in a dataset.

\subsection{Utility Metrics}

We evaluate utility of the model based on the fitness of the model. We use model fitness percentage (MFP) to assess model fitness defined as the normalized root mean squared error (NRMSE) expressed as a percentage ${ }^{17}$. Let $\mathbf{x}$ denote the average time series of a cluster of households and $\mathbf{x}_{\text {model }}$ denote the (in-sample) predicted or the simulated response time-series of the model. Further, let $\overline{\mathbf{x}}$ denote a time series whose elements are equal to the mean of the elements in x. Then

$$
\text { MFP }=100\left(1-\frac{\left\|\mathbf{x}-\mathbf{x}_{\text {model }}\right\|_{2}}{\|\mathbf{x}-\overline{\mathbf{x}}\|_{2}}\right) .
$$

MFP varies between negative infinity to 100 which corresponds to a perfect fit. If $\mathrm{MFP}=0$, then the fitted model is performing no better than the vector $\overline{\mathbf{x}}$, which is simply a straight line equal to the mean of the data at each step.

\section{Adversarial Performance}

In this section, we present our empirical results on the performance of adversary $\mathcal{A}_{2}$ benchmarked against the baseline, i.e., adversary $\mathcal{A}_{1}$. We first demonstrate cases where in contrast to $\mathcal{A}_{1}$, adversary $\mathcal{A}_{2}$ is able to infer daily energy consumption of target households (by predicting daily peaks and troughs of the time series). We then compare the overall mean squared error performance of the two adversaries.

\subsection{Inferring Daily Events}

Figure 3 shows the daily average consumption of the Cluster-1 (62 households) of the UMass-2016 dataset (black line). We also show the daily energy consumption of four households in the cluster (yellow lines). As can be observed, individual consumption can deviate significantly from the average consumption. The same figure also shows the reconstructed daily time-series by $\mathcal{A}_{1}$ (red line). In all cases, the reconstructed time-series is "flat," meaning that with access to only monthly aggregates, the adversary is not able to infer daily consumption with significant precision.

On the other hand, looking at Figure 3 a, we can see that $\mathcal{A}_{2}$, who has access to both model parameters of the cluster average and the monthly aggregates of the target household, can infer daily peaks and troughs for the majority of days

\footnotetext{
${ }^{15}$ See https://au.mathworks.com/help/ident/ref/arx.html

${ }^{16}$ See http: //cvxr.com/cvx/.

${ }^{17}$ This is implemented by the FitPercent method in MATLAB; see https://www.mathworks.com/help/ident/ug/ model-quality-metrics.html.
} 


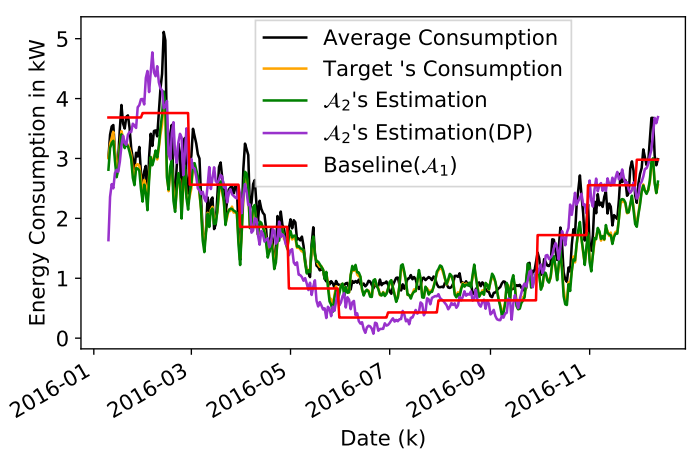

(a) Target Apartment-1

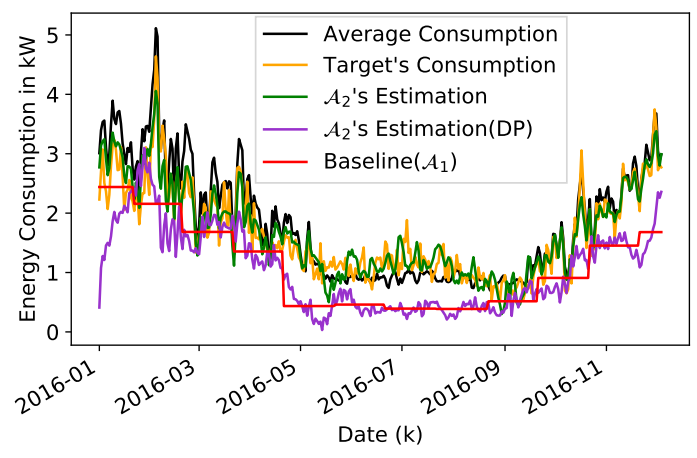

(c) Target Apartment-3

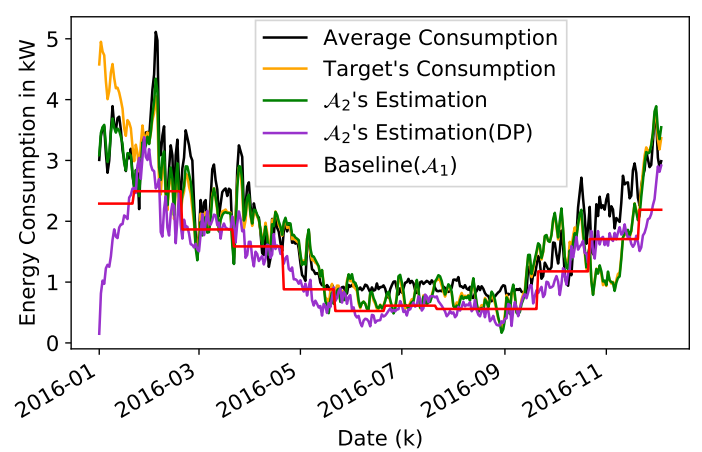

(b) Target Apartment-2

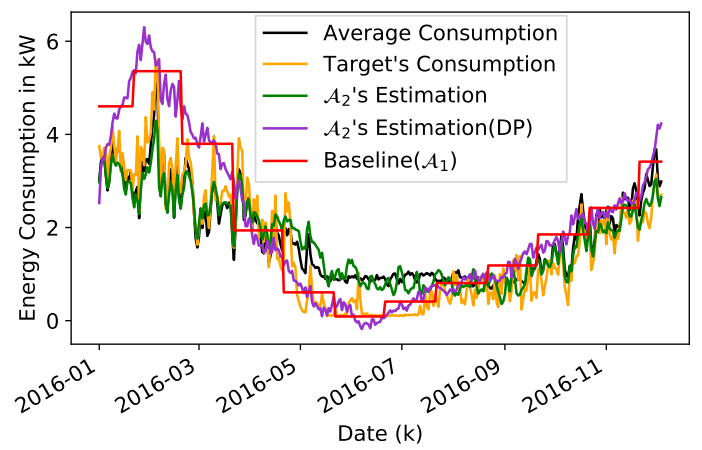

(d) Target Apartment-4

Figure 3: The estimated energy consumption of $\mathcal{A}_{2}$ using raw and perturbed (using LPA with $\epsilon=1$ ) target models compared with the target households' actual energy consumption and the average consumption over all households in Cluster-1 of UMass-2016 dataset.

over the horizon (green line). Note that this is even true where the average cluster time-series deviates from the target household's consumption. As mentioned before, inference to this degree has privacy implications, e.g., the adversary can infer which days the house was not occupied [Molina-Markham et al., 2010]. We do note that $\mathcal{A}_{2}$ is not always able to correctly infer daily consumption as is evident from Figure 3d, where the adversary's inferred time-series is closer to the average time-series than the actual energy consumption of the target household. Nevertheless, this demonstrates that there are households at significant privacy risk if the predictive model of their constituent clusters is released.

\subsection{Evaluating Daily Energy Estimates}

We now evaluate the average performance of the adversaries over all datasets. For quantitative analysis, we plot the empirical cumulative distribution functions (CDFs) of MSE (adversarial error) over all households in clusters of the aforementioned datasets. Each CDF graph contains errors based on three percentiles (abbreviated as Centile in the legends of the graphs): $50 \%$ (median), $75 \%$ and $90 \%$. We benchmark $\mathcal{A}_{1}$ 's performance errors as the baseline (red solid line in the graphs).

UMass Datasets Figures 4 and 5 present performance errors of both adversaries on the two clusters constructed each from the UMass-2015 and the UMass-2016 datasets where MFPs were around 86. We note that the performance errors of both adversaries including the baseline are negligible over time using UMass-2015 dataset (see Figure 4) for both the clusters. Nevertheless, the later adversary (i.e., $\mathcal{A}_{2}$ ) having access to the model parameters performed better than that of the baseline although the performance of the adversary was no better than the baseline until 40th percentile and above 95th percentile. Figure 5 shows that the performance errors of $\mathcal{A}_{2}$ decreased by $67.28 \%, 66.33 \%$ and $63.02 \%$ (i.e. percentage decrease in error) compared to the baseline respectively at 50th, 75th and 90th percentiles using Cluster-1 containing 62 households in UMass-2016 dataset (see Figure 5a). Moreover, using Cluster-2 containing 52 households we observe that the adversary outperformed the baseline and the adversary's performance was comparable 


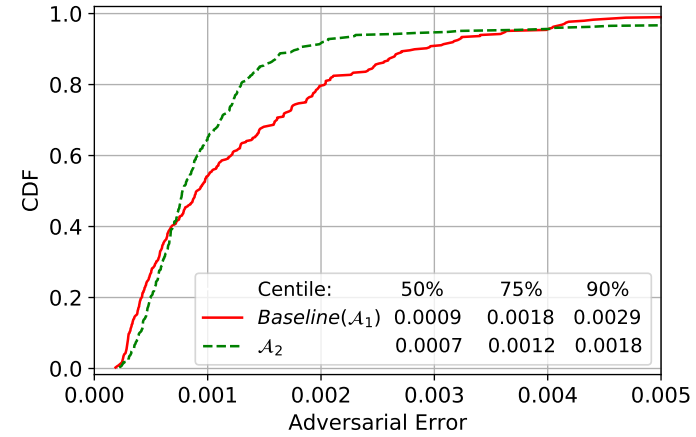

(a) Cluster-1

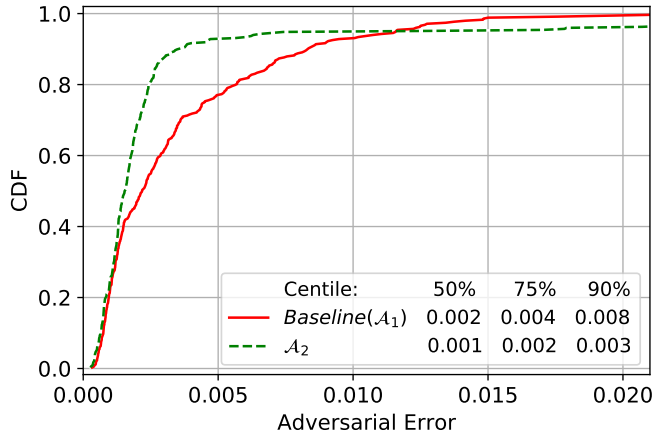

(b) Cluster-2

Figure 4: Performance errors (Mean Squared Errors) of the adversaries using UMass-2015 dataset

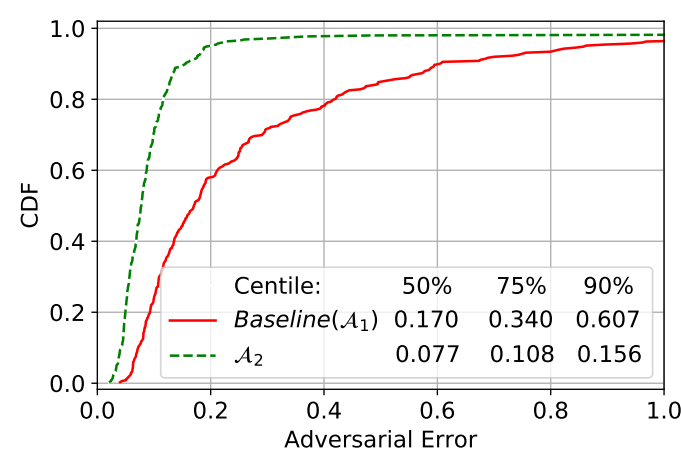

(a) Cluster-1

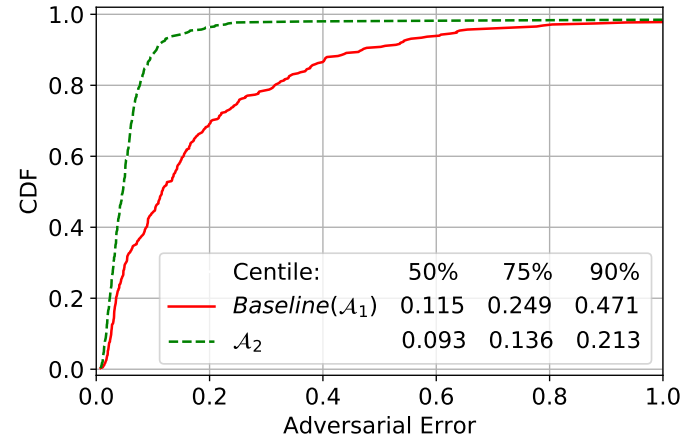

(b) Cluster-2

Figure 5: Performance errors (Mean Squared Errors) of the adversaries using UMass-2016 dataset

to Cluster-1. We observed that the actual average daily consumption of UMass-2015 households was between 0 and $0.30 \mathrm{~kW}$ whereas, the average daily consumption of UMass-2016 households was between 0 and $4 \mathrm{~kW}$ over the year. This indicates that the adversaries may incur lower errors when the daily-to-day consumption are lower and vice-versa.

London Households Dataset We plot the results of only two clusters in Figure 6. We demonstrate results of Cluster1 (Figure 6a) and Cluster-2 (Figure 6b) which contain 936 and 701 households respectively. We observe that $\mathcal{A}_{2}$ outperforms the baseline in both clusters. However, in Cluster-2 the adversary incurred higher errors. We also observe that $\mathcal{A}_{2}$ 's inference performance errors were much higher than the aforementioned datasets although the adversary's overall performance were better than the baseline. We also observed similar behaviour in the adversarial performance in other clusters, due to the space limitation we could not plot all of them. Thus, we observe that the inference performance of the adversaries varies from dataset to dataset and cluster to cluster. We note that MFPs in using the clusters in London-2013 dataset were much lower (between 61 and 72) than that of the UMass datasets (around 86 see Table 2). One of the reasons for this performance variance in different clusters could also be due to the lower Silhouette scores when forming the clusters, high Silhouette score renders good fitness in forming the clusters and vice-versa. We observed that the Silhouette scores were much lower in the clusters on which the adversarial errors were higher.

We observe that the adversary performs better when MFP the ARX model is higher as we found for UMass datasets. We also observed that the day-to-day consumption of London-2013 households were much higher than that of the aforementioned datasets. The higher consumption may also reflect on the performance as we discussed earlier that the adversaries may incur higher errors while the consumption is higher or size of the cluster is very large. Finally, we observe that the adversarial performance deteriorates if the consumption patterns in a cluster are not similar (see Figure 6c). 


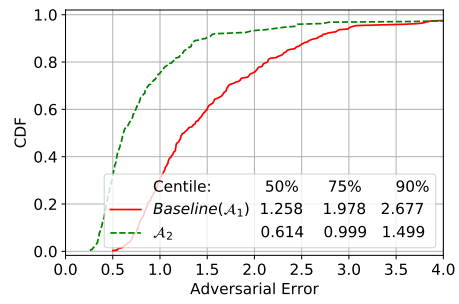

(a) Cluster-1

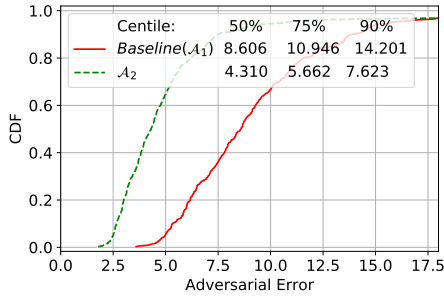

(b) Cluster-2



(c) A Cluster with dissimilar consumption patterns

Figure 6: Performance errors (Mean Squared Errors) of the adversaries using London Smart Meter dataset

\begin{tabular}{lll}
\hline Dataset & Cluster & MFP \\
\hline \multirow{2}{*}{ UMass-2015 } & Cluster-1 & 86.94 \\
& Cluster-2 & 87.36 \\
\multirow{2}{*}{ UMass-2016 } & Cluster-1 & 85.02 \\
& Cluster-2 & 87.27 \\
London-2013 & Cluster-1 & 71.09 \\
& Cluster-2 & 61.79 \\
\hline
\end{tabular}

Table 2: FitPercent of the model (or MFP) on aggregated time-series of each clusters of the datasets.

\section{Evaluating Adversarial Performance under Differential Privacy}

In this section, we evaluate the performance of the adversaries when the ARX model parameters are privacy protected via DP [Dwork et al., 2006b, Dwork and Roth, 2014]. We first present how we obtain the differentially private ARX model and then we evaluate the performance of the adversaries. The goal is to determine the extent to which DP can defend against inferring fine-grained energy data.

\subsection{Applying Differential Privacy}

This brief introduction to DP is tailored to our use case of time-series data. Let $\mathcal{X}$ denote the domain of energy data in a day (e.g., consumption from zero to the maximum consumption during a day in kilowatts). We model a time-series over the horizon $T$ as a $T$-tuple of events. Each event is an element of the domain $\mathcal{X}$, and the domain of the time-series is $\mathcal{X}^{T}$. Our dataset, that is, a collection of $n$ time-series in a cluster, therefore has the domain $\mathcal{X}^{T \times n}$. We say that two datasets $D, D^{\prime} \in \mathcal{X}^{T \times n}$ are neighbours, if they differ in exactly one of the $T \times n$ events.

Definition 1 (Differential Privacy [Dwork et al., 2006b]). A randomized algorithm $\mathcal{M}$ with domain $\mathcal{X}^{T \times n}$ and range Range $(\mathcal{M})$ is said to be $(\epsilon, \delta)$-differentially private iffor all subsets $S$ of Range $(\mathcal{M})$ and for all neighbouring datasets $D, D^{\prime} \in \mathcal{X}^{T \times n}$, we have

$$
\operatorname{Pr}[\mathcal{M}(x) \in S] \leq e^{\epsilon} \operatorname{Pr}\left[\mathcal{M}\left(x^{\prime}\right) \in S\right]+\delta,
$$

where $\epsilon$ is the privacy parameter. If $\delta=0$, we say that $\mathcal{M}$ is $\epsilon$-differentially private [Dwork and Roth, 2014].

Informally, the above definition guarantees that the outcome of the differentially private mechanism will be indistinguishable over datasets in which a single event (one daily reading from one of the households) has been changed. Since we are interested in hiding daily events, we adopt this event-level notion of privacy [Dwork and Roth, 2014]. The ARX model will be trained on the average time-series of a cluster of $n$ households. The average time-series over neighbouring datasets can therefore change by at most $\frac{1}{n} \Delta x$, where $\Delta x$ is called the sensitivity of the summation function, and is defined as

$$
\Delta x=\sup (\mathcal{X})-\inf (\mathcal{X}) .
$$

That is, the maximum possible change in the daily energy consumption. Given $n$ time series $\mathbf{x}_{1}, \mathbf{x}_{2}, \ldots, \mathbf{x}_{n}$, our mechanism to release differentially private ARX model parameters is to apply input perturbation on the average timeseries. More specifically, instead of using the original average time series $\mathbf{x}$ in Eq. (4), we use the perturbed time-series

$$
\tilde{\mathbf{x}}=\left[\begin{array}{llll}
\tilde{x}_{i}(1) & \tilde{x}_{i}(2) & \ldots & \tilde{x}_{i}(T)
\end{array}\right]^{\top},
$$

where $\tilde{x}_{i}(k)=\frac{1}{n}\left(\sum_{i=1}^{n} x_{i}(k)+\eta\right)$ for all $k \in\{1, \ldots, T\}$, and $\eta$ is an i.i.d. sample drawn either from the zero-mean Laplace distribution with scale $\Delta x / \epsilon$ or the zero-mean Gaussian distribution with scale $\frac{\sqrt{2 \ln (2 / \delta)}}{\epsilon} \cdot \Delta x$. 
We call the former, the Laplace perturbation algorithm (LPA) and the latter the Gaussian perturbation algorithm (GPA). With these scales, LPA is $\epsilon$-differentially private [Dwork et al., 2006b] and GPA is $(\epsilon, \delta)$-differentially private [Dwork et al., 2006a] under Definition 1. The perturbed time-series is then used to obtain the ARX model parameters $H$ and $B$ as defined by Eq. (4), which retain DP due to the post-processing property of DP [Dwork and Roth, 2014]. Note that the exogenous input $\mathbf{u}$ and the Gaussian noise $\mathbf{w}$ in the equation are external parameters not dependent on the data of the households.

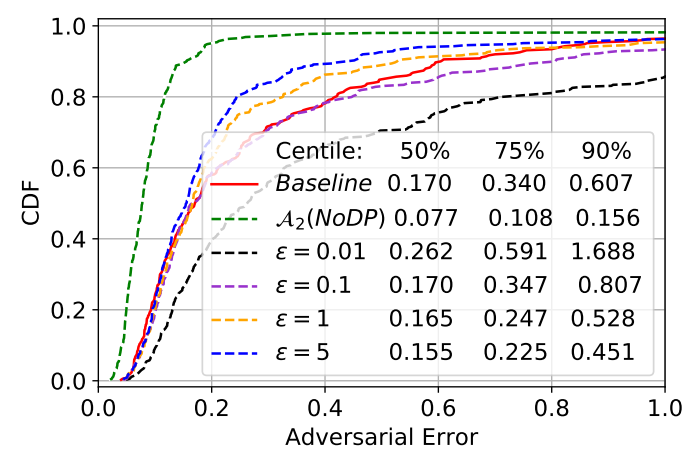

(a) LPA

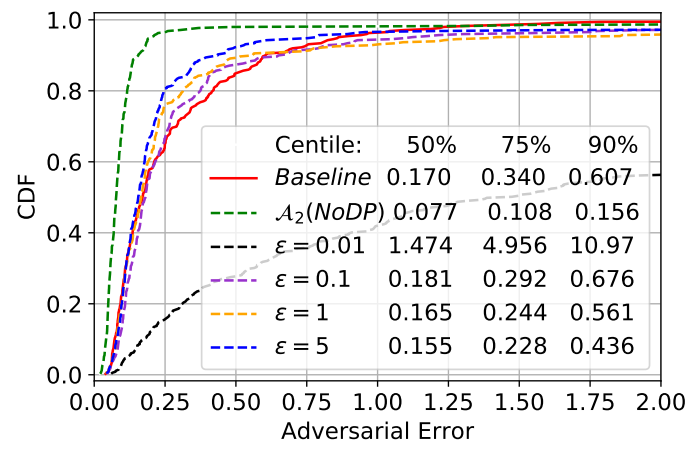

(b) GPA

Figure 7: The performance error of $\mathcal{A}_{2}$ using unperturbed and DP ARX Models with different values of $\epsilon$ based on Cluster-1 in UMass-2016 dataset.

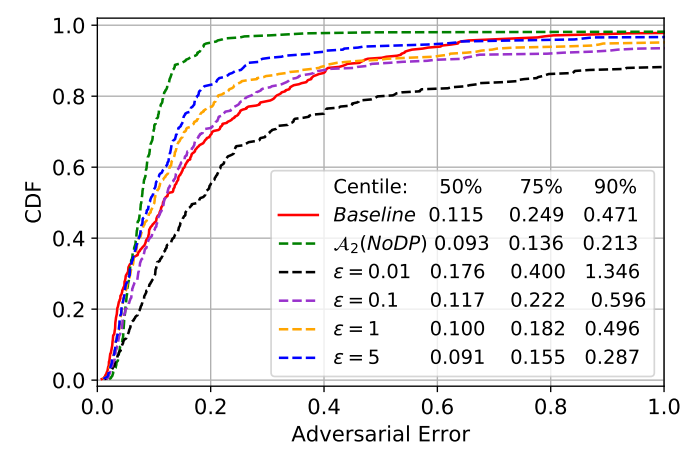

(a) LPA

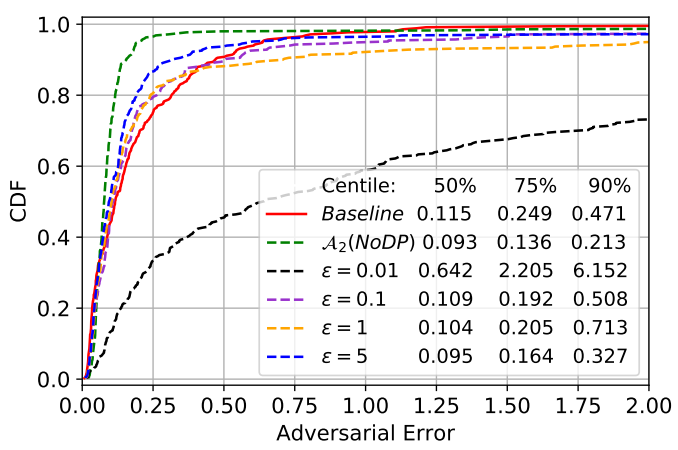

(b) GPA

Figure 8: The performance error of $\mathcal{A}_{2}$ using DP ARX Models with different values of $\epsilon$ based on Cluster-2 in UMass2016 dataset.

\subsection{Experimental Setup}

We compute the sensitivity $\Delta x$ of daily energy consumption time-series by finding the maximum of the difference between two extreme energy consumptions of the individual households for a given dataset. We chose the following values of $\epsilon \in\{0.01,0.1,1,5\}$ for both LPA and GPA, and set $\delta=2^{-20}$ for GPA. We re-train the ARX model using the differentially private time-series $\tilde{\mathbf{x}}$ for the aforementioned various privacy budgets, and release the parameters. The rest of the setup is the same as in Section 3.4. We also note that the $\mathcal{A}_{1}$ (baseline) is given aggregate monthly statistics without any perturbation.

\subsection{Evaluating Adversarial Performance}

In this section, we report adversarial performance in estimating daily energy consumption of the target households using the parameters of the ARX model trained on DP daily consumption data.

UMass-2016 (Cluster-1): We present the results for the UMass-2016 dataset. Figure 7 shows the error CDFs of $\mathcal{A}_{2}$ on UMass-2016 with four different privacy budgets. We observe that for low $\epsilon$ value (at $\epsilon=0.01$ ) LPA and GPA both 


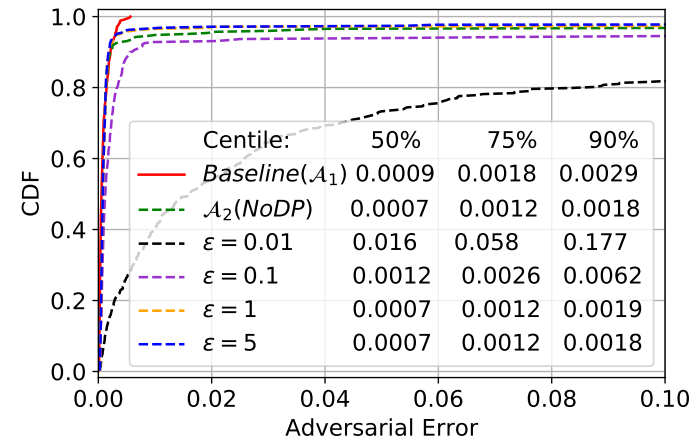

(a) LPA

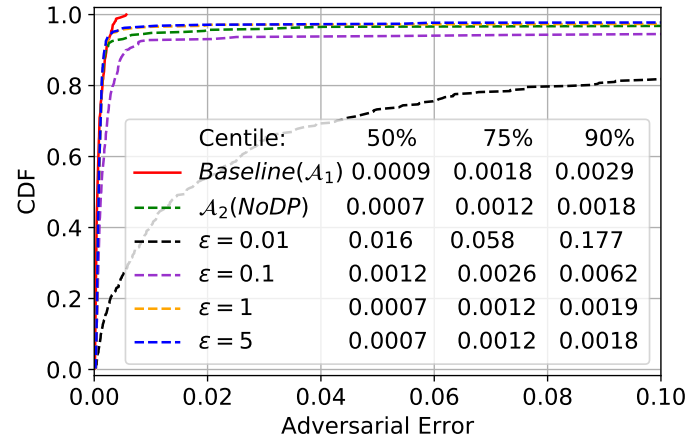

(b) GPA

Figure 9: The performance error of $\mathcal{A}_{2}$ using DP ARX Models with different values of $\epsilon$ based on Cluster-2 in UMass2016 dataset.

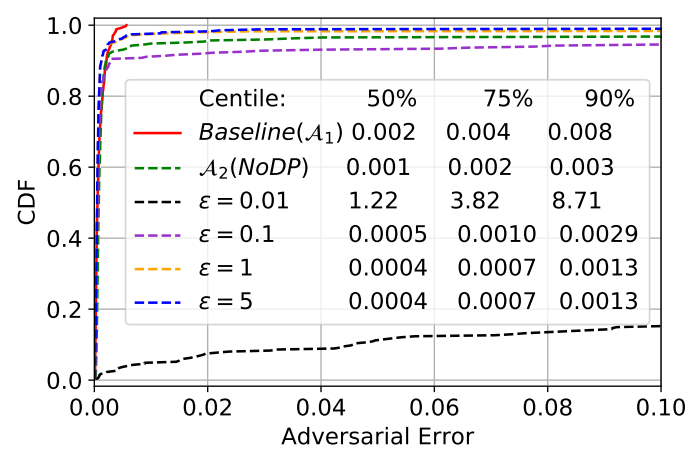

(a) LPA

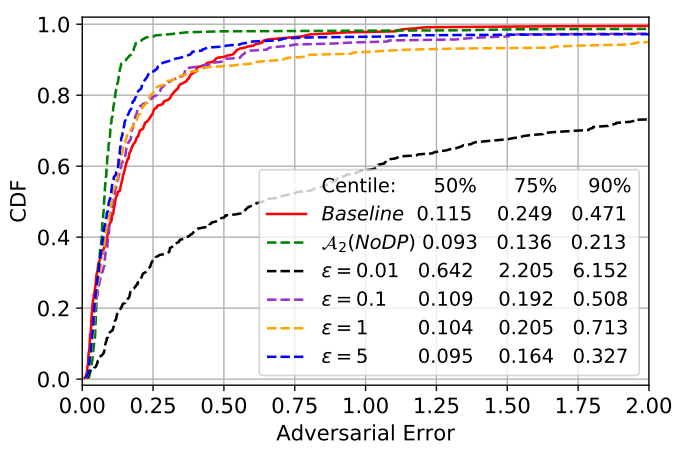

(b) GPA

Figure 10: The performance error of $\mathcal{A}_{2}$ using DP ARX Models with different values of $\epsilon$ based on Cluster-2 in UMass-2016 dataset.

render excellent privacy protection, incurring a huge adversarial error as compared to the baseline in reconstructing fine-grained consumption. Moreover, as we shall discuss in the next section, this protection comes at a high cost to utility. Setting $\epsilon=0.1$ we observe both mechanisms render a good privacy level and incur errors similar to or worse than the baseline. Figure 3 presents the $\mathcal{A}_{2}$ 's estimation (DarkOrchid color graph) when the model was perturbed using LPA at $\epsilon=0.1$. This shows that the adversary's inference capability declined significantly. Nevertheless, this protection comes with a moderate cost to utility. Increasing $\epsilon$ to 1 , we see that the Laplace mechanism does not provide good privacy protection between the 60th and 90th percentiles; however, in the rest of the percentile ranges the adversary's performance was as bad as the baseline. Looking at the graphs in Figure 3, the adversary's predicted time-series is not near the actual time-series or even the average time-series in the first 3 houses. The adversary seems to be performing better in terms of average error, but it does not mean that the adversary is inferring daily events. Overall, we say at $\epsilon=1$ both mechanisms render a moderate privacy guarantee. Further, setting the value of $\epsilon$ to 5 (again, the most relaxed privacy setting considered by us), we observe that the privacy protection rendered by both DP mechanisms is weaker, as the adversary's performance is better than the baseline. We shall see in the next section that, on the other hand, this provides high utility.

UMass-2016 (Cluster-2): We now present the results for the Cluster-2 in UMass-2016 dataset. Figure 8 shows the error CDFs of $\mathcal{A}_{2}$ on Cluster-2 with four different privacy budgets. We observe that for a smaller privacy budget (at $\epsilon=0.01$ ) both mechanisms render excellent privacy protection, incurring a huge adversarial error as compared to the baseline in inferring fine-grained consumption. Moreover, as we shall discuss in the next section, this protection comes at a high cost to utility. Increasing the privacy budget to $\epsilon=0.1$, we observe that both LPA and GPA render a good privacy level and incur errors similar to or worse than the baseline. Finally, we say at $\epsilon=1$ both mechanisms render a moderate privacy guarantee. Further, setting the value of $\epsilon$ to 5 (again, the most relaxed privacy setting considered by us), we observe that the privacy protection rendered by both DP mechanisms is weaker, as the adversary's performance 
is better than the baseline. We shall see in the next section that, on the other hand, this provides high utility. Overall, the performance of the adversary using Cluster-1 is comparable to that of using Cluster-2 in UMass-2016 dataset.

Similarly, Figures $9 \& 10$ show the error CDFs of $\mathcal{A}_{2}$ on two disjoint clusters in UMass-2015 dataset with four different privacy budgets. We observe that for low $\epsilon$ value (at $\epsilon=0.01$ ) LPA and GPA both render excellent privacy protection, incurring a huge adversarial error as compared to the baseline in reconstructing fine-grained consumption. Moreover, as we shall discuss in the next section, this protection comes at a high cost to utility. Setting $\epsilon=0.1$ we observe both mechanisms render good privacy levels incurring errors similar to or worse than the baseline. Overall, we see that both DP mechanisms render a moderate privacy guarantee at $\epsilon=1$. Further, setting the value of $\epsilon$ to 5 (again, the most relaxed privacy setting considered by us), we observe that the privacy protection rendered by both DP mechanisms is weaker, as the adversary's performance is better than the baseline. We shall see in the next section that, on the other hand, this provides high utility.

\begin{tabular}{|c|c|c|c|c|c|}
\hline & $\epsilon$ & 0.01 & 0.1 & 1 & 5 \\
\hline LPA & & -55.63 & 69.53 & 80.69 & 83.87 \\
\hline GPA & & -356.13 & 58.35 & 79.25 & 83.78 \\
\hline
\end{tabular}

Table 3: FitPercent of the perturbed model (or MFP) using Cluster-1 of UMass-2016 dataset at various values of $\epsilon$. Note that the unperturbed model has MFP 86.94.

\begin{tabular}{|c|c|c|c|c|c|}
\hline & $\epsilon$ & 0.01 & 0.1 & 1 & 5 \\
\hline LPA & & -90.00 & 60.35 & 81.65 & 84.95 \\
\hline GPA & & -215.17 & 64.19 & 80.95 & 85.26 \\
\hline
\end{tabular}

Table 4: FitPercent of the model ( or MFP) using Cluster-2 of UMass-2016 dataset at various values of $\epsilon$. Note that the unperturbed model has MFP 85.32

\subsection{Utility}

To obtain the utility of the perturbed model we evaluate the MFP against the unperturbed averaged time-series $\mathbf{x}$. Thus, we replace $\mathbf{x}_{\text {model }}$ in Eq. (11) by $\tilde{\mathbf{x}}_{\text {model }}$ which is the predicted response of the perturbed model.

As discussed in the previous section, the LPA and GPA provide the highest level of protection for the privacy budget at 0.01 (Figure 7). Looking at Table 3, however, this parameter value destroys the utility of the model completely. Setting the privacy budget to 0.1 model utility remains moderate (around $70 \%$ and $60 \%$ for the LPA and GPA respectively) along with good privacy protection (see Table 3). A privacy budget of $\epsilon=1$ still provides privacy guarantee to some extent along with good utility of the model, rather MFP remains close to that of the unperturbed model. Finally, setting the privacy budget to 5 , we note that the utility of the model increased more, however, as mentioned before with $\epsilon=5$ the adversary begins to infer much better than the baseline, and hence the mechanisms only provide weak privacy guarantees. Further, Table 4) shows similar results from Cluster-2 to that the Cluster-1.

UMass-2015 dataset: We now discuss the utility of the models when using the clusters in the UMass-2015 dataset. As discussed in the previous section, the LPA and GPA provide the highest level of protection for the privacy budget at 0.01 (Figure 9). Looking at Table 5, however, this parameter value destroys the utility of the model completely. Setting the privacy budget to 0.1 model utility remains low along with good privacy protection (see Table 5). Note that the GPA provides very low utility when we set the privacy budget at $\epsilon=0.1$. A privacy budget of $\epsilon=1$ still provides a privacy guarantee to some extent along with good utility of the model, rather MFP remains moderate (around $90 \%$ and $50 \%$ for LPA and GPA, respectively) compared to that of the unperturbed model. Finally, setting the privacy budget to 5 , we note that the utility of the model increased more: however, as mentioned before with $\epsilon=5$ the adversary begins to infer much better than the baseline, and hence the mechanisms only provide weak privacy guarantees. Furthermore, Table 6 presents the utility of the models' parameters in terms of model fitness using Cluster- 2 . We observe the utility scores in Cluster-2 dataset is quite similar to that of Cluster-1.

\section{Conclusion}

We studied privacy implications of statistical models trained on fine-grained (i.e., daily) energy data. We empirically evaluated an adversary's performance having access to the model parameters against an adversary (benchmarked as the baseline) who does not have access to the model parameters. We instantiate that the adversaries can use a maximum 


\begin{tabular}{|c|c|c|c|c|c|}
\hline & $\epsilon$ & 0.01 & 0.1 & 1 & 5 \\
\hline LPA & & -210.87 & 22.79 & 71.97 & 76.26 \\
\hline GPA & & -1670.6 & -26.40 & 41.86 & 71.03 \\
\hline
\end{tabular}

Table 5: FitPercent of the perturbed model (or MFP) using Cluster-1 of UMass-2015 dataset at various values of $\epsilon$. Note that the unperturbed model has MFP 77.28 .

\begin{tabular}{|c|c|c|c|c|c|}
\hline & $\epsilon$ & 0.01 & 0.1 & 1 & 5 \\
\hline LPA & & -376.61 & 45.81 & 75.66 & 77.61 \\
\hline GPA & & -675.28 & -88.29 & 59.95 & 75.00 \\
\hline
\end{tabular}

Table 6: FitPercent of the model ( or MFP) using Cluster-2 of UMass-2015 dataset at various values of $\epsilon$. Note that the unperturbed model has MFP 78.15

a posteriori method for reconstructing fine-grained data from the aggregates using two real datasets. We quantify that an adversary infers the fine-grained time-series from the monthly aggregates with high accuracy having access to the model which was trained on average energy consumption time-series data of a cluster of households. We also investigate to what extent DP can mitigate the adversarial threats uncovered in our evaluation. When we applied DP noise to perturb the model, we found that both LPA and GPA mechanisms provide good privacy guarantee against the inference attack at $\epsilon=0.1$ as the adversarial errors were similar to or worse than the baseline. Further, we showed that at this privacy budget the model has moderate utility in terms of model fitness to the cluster.

Overall, our work presents a novel methodology, the MAP estimation equipped to evaluate inference on AR statistical model with aggregate energy-consumption data as prior. Moreover, our methodology can be used to quantify privacy implications in other real-world settings such as location data and transaction data. Our methodology can also be used by the open source and commercial service (SMaaS/MLaaS) providers to appraise privacy protection before releasing the parameters and hyper-parameters of other statistical and ML models, such as Auto-Regressive Moving Average, Vector Auto-Regressive and LSTM trained on fine-grained temporal data.

\section{References}

[ena, 2015] (2015). Energy networks association, "smart meter aggregation assessment final report.

[Ács and Castelluccia, 2011] Ács, G. and Castelluccia, C. (2011). I have a dream!(differentially private smart metering). In International Workshop on Information Hiding, pages 118-132. Springer.

[Alden et al., 2020] Alden, R. E., Gong, H., Ababei, C., and Ionel, D. M. (2020). Lstm forecasts for smart home electricity usage. In 2020 9th International Conference on Renewable Energy Research and Application (ICRERA), pages 434-438. IEEE.

[AlShehhi and Wang, 2020] AlShehhi, M. and Wang, D. (2020). Machine learning pipeline for reusing pretrained models. In Proceedings of the 12th International Conference on Management of Digital EcoSystems, pages 72-75.

[Avsec, 2018] Avsec, Ž. (2018). Kipoi: accelerating the community exchange and reuse of predictive models for genomics. In ICML Workshop for Computational Biology.

[Baloglu and Demir, 2018] Baloglu, U. B. and Demir, Y. (2018). Lightweight privacy-preserving data aggregation scheme for smart grid metering infrastructure protection. International Journal of Critical Infrastructure Protection, 22:16-24.

[Barbosa et al., 2016] Barbosa, P., Brito, A., and Almeida, H. (2016). A technique to provide differential privacy for appliance usage in smart metering. Information Sciences, 370:355-367.

[Barbosa et al., 2014] Barbosa, P., Brito, A., Almeida, H., and Clauß, S. (2014). Lightweight privacy for smart metering data by adding noise. In Proceedings of the 29th Annual ACM Symposium on Applied Computing, pages 531-538.

[Beckel et al., 2014] Beckel, C., Sadamori, L., Staake, T., and Santini, S. (2014). Revealing household characteristics from smart meter data. Energy, 78:397-410.

[Bohli et al., 2010] Bohli, J.-M., Sorge, C., and Ugus, O. (2010). A privacy model for smart metering. In 2010 IEEE International Conference on Communications Workshops, pages 1-5. IEEE.

[Box et al., 2015] Box, G. E., Jenkins, G. M., Reinsel, G. C., and Ljung, G. M. (2015). Time series analysis: forecasting and control. John Wiley \& Sons.

[Buescher et al., 2017] Buescher, N., Boukoros, S., Bauregger, S., and Katzenbeisser, S. (2017). Two is not enough: Privacy assessment of aggregation schemes in smart metering. Proceedings on Privacy Enhancing Technologies, 2017(4):198-214. 
[Chatzikokolakis et al., 2013] Chatzikokolakis, K., Andrés, M. E., Bordenabe, N. E., and Palamidessi, C. (2013). Broadening the scope of differential privacy using metrics. In International Symposium on Privacy Enhancing Technologies Symposium, pages 82-102. Springer.

[Chen et al., 2015] Chen, D., Kalra, S., Irwin, D., Shenoy, P., and Albrecht, J. (2015). Preventing occupancy detection from smart meters. IEEE Transactions on Smart Grid, 6(5):2426-2434.

[Dagnely et al., 2015] Dagnely, P., Ruette, T., Tourwé, T., Tsiporkova, E., and Verhelst, C. (2015). Predicting hourly energy consumption. can regression modeling improve on an autoregressive baseline? In International Workshop on Data Analytics for Renewable Energy Integration, pages 105-122. Springer.

[Dambreville et al., 2014] Dambreville, R., Blanc, P., Chanussot, J., and Boldo, D. (2014). Very short term forecasting of the global horizontal irradiance using a spatio-temporal autoregressive model. Renewable Energy, 72:291-300.

[Danezis et al., 2011] Danezis, G., Kohlweiss, M., and Rial, A. (2011). Differentially private billing with rebates. In International Workshop on Information Hiding, pages 148-162. Springer.

[Dwork, 2011] Dwork, C. (2011). Differential privacy. Encyclopedia of Cryptography and Security, pages 338-340.

[Dwork et al., 2006a] Dwork, C., Kenthapadi, K., McSherry, F., Mironov, I., and Naor, M. (2006a). Our data, ourselves: Privacy via distributed noise generation. In Annual International Conference on the Theory and Applications of Cryptographic Techniques, pages 486-503. Springer.

[Dwork et al., 2006b] Dwork, C., McSherry, F., Nissim, K., and Smith, A. (2006b). Calibrating noise to sensitivity in private data analysis. In Theory of cryptography conference, pages 265-284. Springer.

[Dwork and Roth, 2014] Dwork, C. and Roth, A. (2014). The algorithmic foundations of differential privacy. Foundations and Trends in Theoretical Computer Science, 9(3-4):211-407.

[Dwork et al., 2017] Dwork, C., Smith, A., Steinke, T., and Ullman, J. (2017). Exposed! a survey of attacks on private data. Annual Review of Statistics and Its Application, 4:61-84.

[Eibl and Engel, 2014] Eibl, G. and Engel, D. (2014). Influence of data granularity on smart meter privacy. IEEE Transactions on Smart Grid, 6(2):930-939.

[Eibl and Engel, 2017] Eibl, G. and Engel, D. (2017). Differential privacy for real smart metering data. Computer ScienceResearch and Development, 32(1-2):173-182.

[Erkin et al., 2013] Erkin, Z., Troncoso-Pastoriza, J. R., Lagendijk, R. L., and Pérez-González, F. (2013). Privacy-preserving data aggregation in smart metering systems: An overview. IEEE Signal Processing Magazine, 30(2):75-86.

[Fredrikson et al., 2015] Fredrikson, M., Jha, S., and Ristenpart, T. (2015). Model inversion attacks that exploit confidence information and basic countermeasures. In Proceedings of the 22nd ACM SIGSAC Conference on Computer and Communications Security, pages 1322-1333.

[Fredrikson et al., 2014] Fredrikson, M., Lantz, E., Jha, S., Lin, S., Page, D., and Ristenpart, T. (2014). Privacy in pharmacogenetics: An end-to-end case study of personalized warfarin dosing. In 23rd \{USENIX\} Security Symposium ( $\{$ USENIX\} Security 14), pages 17-32.

[Galrinho et al., 2017] Galrinho, M., Everitt, N., and Hjalmarsson, H. (2017). Arx modeling of unstable linear systems. Automatica, 75:167-171.

[Gauvain and Lee, 1994] Gauvain, J.-L. and Lee, C.-H. (1994). Maximum a posteriori estimation for multivariate gaussian mixture observations of markov chains. IEEE Transactions on Speech and Audio Processing, 2(2):291-298.

[Giaconi et al., 2020] Giaconi, G., Gunduz, D., and Poor, H. V. (2020). Smart meter data privacy. arXiv preprint arXiv:2009.01364.

[Green et al., 2014] Green, R., Staffell, I., and Vasilakos, N. (2014). Divide and conquer? k-means clustering of demand data allows rapid and accurate simulations of the british electricity system. IEEE Transactions on Engineering Management, 61(2):251-260.

[Guefano et al., 2021] Guefano, S., Tamba, J. G., Azong, T. E. W., and Monkam, L. (2021). Forecast of electricity consumption in the cameroonian residential sector by grey and vector autoregressive models. Energy, 214:118791.

[Guo et al., 2014] Guo, Z., Wang, Z. J., and Kashani, A. (2014). Home appliance load modeling from aggregated smart meter data. IEEE Transactions on power systems, 30(1):254-262.

[Hayes et al., 2019] Hayes, J., Melis, L., Danezis, G., and De Cristofaro, E. (2019). Logan: Membership inference attacks against generative models. Proceedings on Privacy Enhancing Technologies, 2019(1):133-152.

[Hedayati, 2012] Hedayati, A. (2012). An analysis of identity theft: Motives, related frauds, techniques and prevention. Journal of Law and Conflict Resolution, 4(1):1-12.

[Hossain et al., 2019] Hossain, M. M., Zhang, T., and Ardakanian, O. (2019). Evaluating the feasibility of reusing pre-trained thermal models in the residential sector. In Proceedings of the 1st ACM International Workshop on Urban Building Energy Sensing, Controls, Big Data Analysis, and Visualization, pages 23-32.

[Hotho et al., 2002] Hotho, A., Maedche, A., and Staab, S. (2002). Ontology-based text document clustering. KI, 16(4):48-54. 
[Ji et al., 2018] Ji, Y., Zhang, X., Ji, S., Luo, X., and Wang, T. (2018). Model-reuse attacks on deep learning systems. In Proceedings of the 2018 ACM SIGSAC Conference on Computer and Communications Security, pages 349-363.

[Karim et al., 2020] Karim, F., Majumdar, S., and Darabi, H. (2020). Adversarial attacks on time series. IEEE transactions on pattern analysis and machine intelligence.

[Kaytez, 2020] Kaytez, F. (2020). A hybrid approach based on autoregressive integrated moving average and least-square support vector machine for long-term forecasting of net electricity consumption. Energy, 197:117200.

[Kelly and Knottenbelt, 2015] Kelly, J. and Knottenbelt, W. (2015). Neural nilm: Deep neural networks applied to energy disaggregation. In Proceedings of the 2nd ACM International Conference on Embedded Systems for Energy-Efficient Built Environments, pages 55-64.

[Knirsch et al., 2016] Knirsch, F., Eibl, G., and Engel, D. (2016). Error-resilient masking approaches for privacy preserving data aggregation. IEEE Transactions on Smart Grid, 9(4):3351-3361.

[Kong et al., 2016] Kong, W., Dong, Z. Y., Ma, J., Hill, D. J., Zhao, J., and Luo, F. (2016). An extensible approach for non-intrusive load disaggregation with smart meter data. IEEE Transactions on Smart Grid, 9(4):3362-3372.

[Liao et al., 2014] Liao, X., Formby, D., Day, C., and Beyah, R. A. (2014). Towards secure metering data analysis via distributed differential privacy. In 2014 44th Annual IEEE/IFIP International Conference on Dependable Systems and Networks, pages 780-785. IEEE.

[Liao et al., 2017] Liao, X., Srinivasan, P., Formby, D., and Beyah, A. R. (2017). Di-prida: differentially private distributed load balancing control for the smart grid. IEEE Transactions on Dependable and Secure Computing.

[Lisovich et al., 2010] Lisovich, M. A., Mulligan, D. K., and Wicker, S. B. (2010). Inferring personal information from demandresponse systems. IEEE Security \& Privacy, 8(1):11-20.

[Mak et al., 2019] Mak, T. W., Fioretto, F., Shi, L., and Van Hentenryck, P. (2019). Privacy-preserving power system obfuscation: A bilevel optimization approach. IEEE Transactions on Power Systems.

[Makonin et al., 2015] Makonin, S., Popowich, F., Bajić, I. V., Gill, B., and Bartram, L. (2015). Exploiting hmm sparsity to perform online real-time nonintrusive load monitoring. IEEE Transactions on Smart Grid, 7(6):2575-2585.

[McDougal et al., 2017] McDougal, R. A., Morse, T. M., Carnevale, T., Marenco, L., Wang, R., Migliore, M., Miller, P. L., Shepherd, G. M., and Hines, M. L. (2017). Twenty years of modeldb and beyond: building essential modeling tools for the future of neuroscience. Journal of computational neuroscience, 42(1):1-10.

[McLaughlin et al., 2011] McLaughlin, S., McDaniel, P., and Aiello, W. (2011). Protecting consumer privacy from electric load monitoring. In Proceedings of the 18th ACM conference on Computer and communications security, pages 87-98.

[Molina-Markham et al., 2010] Molina-Markham, A., Shenoy, P., Fu, K., Cecchet, E., and Irwin, D. (2010). Private memoirs of a smart meter. In Proceedings of the 2nd ACM workshop on Embedded Sensing Systems for Energy-Efficiency in Building, pages 61-66. ACM.

[Nasr et al., 2018] Nasr, M., Shokri, R., and Houmansadr, A. (2018). Comprehensive privacy analysis of deep learning: Standalone and federated learning under passive and active white-box inference attacks.

[Nichiforov et al., 2017] Nichiforov, C., Stamatescu, I., Făgărăşan, I., and Stamatescu, G. (2017). Energy consumption forecasting using arima and neural network models. In 2017 5th International Symposium on Electrical and Electronics Engineering (ISEEE), pages 1-4. IEEE.

[Paparoditis and Politis, 2018] Paparoditis, E. and Politis, D. N. (2018). The asymptotic size and power of the augmented dickeyfuller test for a unit root. Econometric Reviews, 37(9):955-973.

[Papernot et al., 2017] Papernot, N., McDaniel, P., Goodfellow, I., Jha, S., Celik, Z. B., and Swami, A. (2017). Practical blackbox attacks against machine learning. In Proceedings of the 2017 ACM on Asia conference on computer and communications security, pages 506-519.

[Philpott, 2006] Philpott, A. (2006). Identity theft-dodging the own-goals. Network Security, 2006(1):11-13.

[Pyrgelis et al., 2017] Pyrgelis, A., Troncoso, C., and De Cristofaro, E. (2017). Knock knock, who's there? membership inference on aggregate location data. arXiv preprint arXiv:1708.06145.

[Quinn, 2009] Quinn, E. L. (2009). Privacy and the new energy infrastructure. Available at SSRN 1370731.

[Ruiz et al., 2016] Ruiz, L. G. B., Cuéllar, M. P., Calvo-Flores, M. D., and Jiménez, M. D. C. P. (2016). An application of nonlinear autoregressive neural networks to predict energy consumption in public buildings. Energies, 9(9):684.

[Sablayrolles et al., 2019] Sablayrolles, A., Douze, M., Schmid, C., Ollivier, Y., and Jégou, H. (2019). White-box vs black-box: Bayes optimal strategies for membership inference. In International Conference on Machine Learning, pages 5558-5567. PMLR.

[Saputro and Akkaya, 2014] Saputro, N. and Akkaya, K. (2014). On preserving user privacy in smart grid advanced metering infrastructure applications. Security and Communication Networks, 7(1):206-220.

[Shah et al., 2020] Shah, I., Bibi, H., Ali, S., Wang, L., and Yue, Z. (2020). Forecasting one-day-ahead electricity prices for italian electricity market using parametric and nonparametric approaches. IEEE Access, 8:123104-123113. 
[Shokri et al., 2017] Shokri, R., Stronati, M., Song, C., and Shmatikov, V. (2017). Membership inference attacks against machine learning models. In 2017 IEEE Symposium on Security and Privacy (SP), pages 3-18. IEEE.

[Singh and Pozo, 2019] Singh, B. and Pozo, D. (2019). A guide to solar power forecasting using arma models. In 2019 IEEE PES Innovative Smart Grid Technologies Europe (ISGT-Europe), pages 1-4. IEEE.

[Tudor et al., 2013] Tudor, V., Almgren, M., and Papatriantafilou, M. (2013). Analysis of the impact of data granularity on privacy for the smart grid. In Proceedings of the 12th ACM Workshop on Privacy in the Electronic Society, pages 61-70. ACM.

[Van Rijn et al., 2013] Van Rijn, J. N., Bischl, B., Torgo, L., Gao, B., Umaashankar, V., Fischer, S., Winter, P., Wiswedel, B., Berthold, M. R., and Vanschoren, J. (2013). Openml: A collaborative science platform. In Joint european conference on machine learning and knowledge discovery in databases, pages 645-649. Springer.

[Vu et al., 2019] Vu, D. H., Muttaqi, K. M., Agalgaonkar, A. P., and Bouzerdoum, A. (2019). Short-term forecasting of electricity spot prices containing random spikes using a time-varying autoregressive model combined with kernel regression. IEEE Transactions on Industrial Informatics, 15(9):5378-5388.

[Wu et al., 2020] Wu, H., Wang, C., Nock, R., Wang, W., Yin, J., Lu, K., and Zhu, L. (2020). Smint: Toward interpretable and robust model sharing for deep neural networks. ACM Transactions on the Web (TWEB), 14(3):1-28.

[Wu et al., 2016] Wu, X., Fredrikson, M., Jha, S., and Naughton, J. F. (2016). A methodology for formalizing model-inversion attacks. In 2016 IEEE 29th Computer Security Foundations Symposium (CSF), pages 355-370. IEEE.

[Xiao et al., 2018] Xiao, J., Li, Y., Xie, L., Liu, D., and Huang, J. (2018). A hybrid model based on selective ensemble for energy consumption forecasting in china. Energy, 159:534-546.

[Yang et al., 2017] Yang, Z., Cheng, P., and Chen, J. (2017). Differential-privacy preserving optimal power flow in smart grid. IET Generation, Transmission \& Distribution, 11(15):3853-3861.

[Yang et al., 2020] Yang, Z., Shao, B., Xuan, B., Chang, E.-C., and Zhang, F. (2020). Defending model inversion and membership inference attacks via prediction purification. arXiv preprint arXiv:2005.03915.

[Zhao et al., 2014] Zhao, J., Jung, T., Wang, Y., and Li, X. (2014). Achieving differential privacy of data disclosure in the smart grid. In IEEE INFOCOM 2014-IEEE Conference on Computer Communications, pages 504-512. IEEE.

[Zhao et al., 2018] Zhao, S., Talasila, M., Jacobson, G., Borcea, C., Aftab, S. A., and Murray, J. F. (2018). Packaging and sharing machine learning models via the acumos ai open platform. In 2018 17th IEEE International Conference on Machine Learning and Applications (ICMLA), pages 841-846. IEEE. 


\section{University Library}

\section{- M M N E R VA A gateway to Melbourne's research publications}

Minerva Access is the Institutional Repository of The University of Melbourne

Author/s:

Sheikh, NU;Asghar, HJ;Farokhi, F;Kaafar, MA

Title:

Do Auto-Regressive Models Protect Privacy? Inferring Fine-Grained Energy Consumption From Aggregated Model Parameters

Date:

2022-11-01

Citation:

Sheikh, N. U., Asghar, H. J., Farokhi, F. \& Kaafar, M. A. (2022). Do Auto-Regressive Models Protect Privacy? Inferring Fine-Grained Energy Consumption From Aggregated Model Parameters. IEEE TRANSACTIONS ON SERVICES COMPUTING, 15 (6), pp.3198-3209. https://doi.org/10.1109/TSC.2021.3100498.

Persistent Link:

http://hdl.handle.net/11343/281338 